\title{
Proof of the Generalized Second LaW FOR Quasistationary SEMICLASSiCAL BLACK HOLES*
}

\author{
VALERY P. FROLOV ${ }^{\dagger \dagger}$ \\ and \\ DON N. PAGE ${ }^{\S}$ \\ CIAR Cosmology Program \\ Theoretical Physics Institute \\ University of Alberta, Edmonton, Canada T6G 2J1
}

1993 Feb. 8; revised Aug. 17

PACS Indices: 97.60.Lf; 05.30.-d; 04.20.Cv

\begin{abstract}
A simple direct explicit proof of the generalized second law of black hole thermodynamics is given for a quasistationary semiclassical black hole.
\end{abstract}

*Alberta-Thy-10-93, gr-qc/9302017

${ }^{\dagger}$ On leave of absence from the P. N. Lebedev Physical Institute, Leninsky prospect 53, Moscow 117924, Russia

‡Internet address: frolov@phys.ualberta.ca

§Internet address: don@page.phys.ualberta.ca 
According to the thermodynamical analogy in black holes physics, the entropy of a black hole in general relativity is defined as $S_{B H}=A / 4$, where $A$ is the area of the black hole surface. Bekenstein [1] made an assumption that the generalized entropy $\widetilde{S}$, i.e. the sum of the black hole entropy $S_{B H}$ and the entropy $S_{\text {rad }}$ of the usual matter and gravitational radiation outside a black hole $\left(\widetilde{S}=S_{B H}+S_{r a d}\right)$, never decreases. This assumption, known as the generalized second law, plays a fundamental role in black hole physics. Though a simple explicit general proof of this law has not been given until now, concrete processes were considered, and the validity of the generalized second law for these concrete processes was verified. (For a discussion of the generalized second law in black hole physics, see, e.g. [2, [3] and references therein.)

A special particular example is the case of an evaporating black hole. Quantum radiation created by a black hole carries entropy to infinity, while the area of the black hole (and hence its entropy) is decreasing. The validity of the generalized second law for the massless radiation evaporated by an uncharged, nonrotating semiclassical black hole was almost proved by Zurek [⿴囗⿴囗十). In particular, he estimated that $R \equiv d S_{\text {rad }} / d S_{B H} \approx \frac{4}{3}$ for the evaporation of a black hole in vacuum. (More accurate numerical calculations of the emission of neutrinos, photons, and gravitons actually had given even higher values [5, 6].) The evaporation of charged black holes was discussed by Schumacher [7].

Unruh and Wald [8] stressed the importance of the vacuum polarization and acceleration radiation effects for the validity of the generalized second law. More general arguments for the validity of this law for slowly evolving black holes were given by Zurek and Thorne [9] and by Thorne, Zurek, and Price [10] in the framework of the membrane paradigm. The latter approach, using the notion of the thermal atmosphere of a black hole, looks quite general and attractive, but it requires such an operation as the renormalization of the entropy density near a black hole. The rigorous foundation of this operation is not completely clear [2], though it can be argued that the renormalization should not affect the derivation for quasistationary changes [11.

The aim of this paper is to present a simple explicit proof of the generalized second law for quasistationary changes of a generic charged rotating black hole emitting, absorbing, and scattering any sort of radiation in the Hawking semiclassical formalism (quantum radiation fields in the classical spacetime background of a black hole whose conserved quantities change by the expectation values of the flux of radiation out of or into it). This proof may be considered to be a mathematical fleshing out of some of the verbal arguments of Zurek, Thorne, 
and Price [9, 10].

A quasistationary black hole may be considered to emit a density matrix $\rho_{0}$ of thermal radiation [12] in UP modes [10]. Suppose there is also radiation with density matrix $\rho_{1}$ incident on the hole from far away (e.g., past null infinity, $\mathcal{I}^{-}$) in the corresponding $I N$ modes (i.e., incoming modes which are of positive frequency at $\mathcal{I}^{-}$). We use the semiclassical approximation and assume that the radiation in these two sets of modes will be quantum mechanically uncorrelated, giving an initial product state

$$
\rho_{\text {initial }} \equiv \rho_{01}=\rho_{0} \otimes \rho_{1}
$$

This assumption is natural for an eternal black hole. For it, the UP modes (defined to be of positive frequencies with respect to the Killing vector null at and tangent to the past horizon, $H^{-}$) vanish at $\mathcal{I}^{-}$, whereas the $I N$ modes vanish at $H^{-}$, and $\mathcal{I}^{-}$and $H^{-}$are causally disconnected.

In the case in which the black hole arises from gravitational collapse and becomes quasistationary, the $U P$ modes are defined to be the same in the future stationary region as the UP modes of the eternal black hole with the same future stationary region. They are nonvanishing at $\mathcal{I}^{-}$at the advanced time at which the black hole forms. One might therefore worry that these modes in principle could be correlated with the $I N$ modes which come from $\mathcal{I}^{-}$at much later advanced time. However, after the hole has become quasistationary, the relevant UP modes trace back to such high energy modes at $\mathcal{I}^{-}$that the state in those modes must be extremely close to the vacuum there. Thus, in our quasistationary approximation, they will have totally negligible correlations with the $I N$ modes coming in much later in advanced time. That is why, for the physics of the quasistationary region at late time, both of the pictures (eternal black hole and black hole arising from collapse) give very nearly the same results. For concreteness, we shall use the eternal black hole picture in the following discussion.

After the initial state $\rho_{01}$ interacts with the classical angular momentum and curvature barrier separating the horizon from infinity, and possibly interacts with itself as well, it will have evolved unitarily into a (generally) correlated final state

$$
\rho_{\text {final }} \equiv \rho_{23} \neq \rho_{2} \otimes \rho_{3}
$$

where

$$
\rho_{2}=\operatorname{tr}_{3} \rho_{23}
$$


is the density matrix of the radiation in the OUT modes escaping to future null infinity $\mathcal{I}^{+}$, and

$$
\rho_{3}=\operatorname{tr}_{2} \rho_{23}
$$

is that in the DOWN modes that are swallowed by the future horizon $\mathrm{H}^{+}$.

The entropy of each of these states is

$$
S_{i}=-\operatorname{tr}\left(\rho_{i} \ln \rho_{i}\right)
$$

Because the evolution from $\rho_{01}$ to $\rho_{23}$ is unitary, $S_{23}=S_{01}$. Furthermore, since $\rho_{01}$ is uncorrelated but $\rho_{23}$ is generically partially correlated, the entropies of these states obey the inequality [13]

$$
S_{2}+S_{3} \geq S_{23}=S_{01}=S_{0}+S_{1}
$$

The first law of black hole physics [14] for a black hole of mass $M$, angular momentum $J$, charge $Q$ (its conserved extensive quantities), and Hawking temperature $T_{B H}$, angular velocity $\Omega$, and electrostatic potential $\Phi$ (its thermodynamically conjugate intensive quantities) implies that

$$
d S_{B H}=T_{B H}^{-1}(d M-\Omega d J-\Phi d Q)=T^{-1} d E
$$

where $T$ and $E$ are the local temperature and energy as measured by a fiducial observer (FIDO) [10] corotating with the hole near the horizon. If $E_{0}$ and $E_{3}$ are the expectation values of the local energies of the emitted state $\rho_{0}$ and the absorbed state $\rho_{3}$, respectively, then the semiclassical approximation, combined with the first law, says that

$$
\Delta S_{B H}=T^{-1}\left(E_{3}-E_{0}\right)
$$

assuming that the changes to the hole are sufficiently small that $T$ stays nearly constant throughout the process (the quasistationary approximation).

Equation (8) and inequality (6) imply that the change in the generalized entropy is

$$
\begin{aligned}
\Delta \widetilde{S} & =\Delta S_{B H}+\Delta S_{r a d}=T^{-1}\left(E_{3}-E_{0}\right)+S_{2}-S_{1} \\
& \geq\left(S_{0}-T^{-1} E_{0}\right)-\left(S_{3}-T^{-1} E_{3}\right)
\end{aligned}
$$

Now for fixed $T$ and for equivalent quantum systems (as the $U P$ modes of $\rho_{0}$ and the corresponding $D O W N$ modes of $\rho_{3}$ are by $C P T$ reversal, for a quasistationary 
black hole), $S-T^{-1} E$ is a Massieu function [15, 16] (essentially the negative of the local free energy divided by the temperature) which is maximized for the thermal state $\rho_{0}$. Therefore,

$$
\Delta \widetilde{S} \geq 0,
$$

which is the generalized second law. This is an explicit mathematical demonstration of what Zurek, Thorne, and Price [9, 10] argued verbally, that the generalized second law is a special case of the ordinary second law, with the black hole as a hot, rotating, charged body that emits thermal radiation uncorrelated with what is incident upon it.

This proof applies to any emission, scattering, or absorption process, even for interacting fields (e.g., for boxes lowered to mine a black hole [8], considered as special IN states, not as changes in the boundary conditions), so long as the semiclassical approximation applies, and so long as the changes take place in a quasistationary manner so that, for example, Eq. (8) is valid. One would conjecture that the generalized second law applies also for rapid changes to a black hole [10], but then $S_{B H}$, one-quarter of the horizon area, would depend upon the future evolution. One would presumably also need to include matter near the hole in $S_{\text {rad }}$, but it is problematic how to do that in a precise way without getting divergences from infinitely short wavelength modes if there is to be a sharp cutoff to exclude matter inside the hole [17, 18]. In a quasistationary process, one can with negligible error allow enough time for the modes to propagate far from the hole, where the states $\rho_{1}$ and $\rho_{2}$ and their respective entropies can be evaluated unambiguously.

On the other hand, it is a controversial open question whether the generalized second law applies for the fine-grained radiation entropy $S_{\text {rad }}=-\operatorname{tr}(\rho \ln \rho)$ outside of the semiclassical approximation [19]. There an enormous number of (individually very small) nonthermal multimode correlations in $\rho_{0}$, perhaps induced at least in part by the quantum back-reaction of the metric, might conceivably restore the full information of the initial collapse state to the final state of the radiation after the hole evaporates away [20, 21, 22, 23]. In other words, one might start off with a pure initial state in which $\widetilde{S}=S_{\text {rad }}=0$, evolve unitarily to form a black hole state in which both $S_{B H}$ and $S_{\text {rad }}$ are positive (from the coarsegraining of dividing the total pure state system into black hole and radiation subsystems and then adding the separate entropy of each, ignoring their mutual correlations), and then go back to another pure radiation state with $\widetilde{S}=S_{\text {rad }}=0$ after the hole disappears and there is no more coarse graining in the definition of 
$\widetilde{S}$.

To illustrate the proof of the generalized second law above, consider the case in which $\rho_{0}, \rho_{1}, \rho_{2}$, and $\rho_{3}$ are the respective density matrices for single $U P$, $I N, O U T$, and DOWN modes that are corresponding in the following sense: Each mode is that of the same free quantum field (the same particle-antiparticle species, assumed to interact only with the semiclassical gravitational and electromagnetic fields of the black hole) and has the same helicity and the same total and orbital angular momentum quantum numbers $j$ and $l$. The $I N$ and OUT modes each have the same positive frequency $\omega>0$ at past and future null infinities, the same azimuthal angular momentum quantum number $m$, and the same charge $q$ (i.e., excitations of both are either particles or antiparticles of the quantum field). The $U P$ and $D O W N$ modes each have the same positive frequency

$$
\widetilde{\omega}=|\omega-m \Omega-q \Phi| \equiv \eta(\omega-m \Omega-q \Phi)=\bar{\omega}-\bar{m} \Omega-\bar{q} \Phi>0
$$

with respect to the Killing vector null at and tangent to the past and future horizons, the same frequency-at-infinity $\bar{\omega}=\eta \omega$, the same azimuthal angular momentum $\bar{m}=\eta m$, and the same charge $\bar{q}=\eta q$, where

$$
\eta=\operatorname{sgn}(\omega-m \Omega-q \Phi)
$$

If $\eta=+1$, any three of the four modes are linearly related. For superradiant modes (bosonic modes with $\eta=-1$ ), the $U P$ and $D O W N$ modes must be complex conjugated before any three of the four modes are linearly related.

The expected numbers of particles $n_{i}$ in the states $\rho_{i}$ are related as follows:

$$
n_{0}-n_{3}=\eta\left(n_{2}-n_{1}\right)
$$

The change in the generalized entropy due to the absorption and emission in these modes is, using the inequality (6),

$$
\Delta \widetilde{S}=S_{2}-S_{1}-x\left(n_{0}-n_{3}\right) \geq S_{0}-S_{3}-x\left(n_{0}-n_{3}\right),
$$

where $x=T_{B H}^{-1} \widetilde{\omega}=T_{B H}^{-1}|\omega-m \Omega-q \Phi|$.

The matrix elements of $\rho_{0}$ in a Fock number basis in the Hilbert space of $U P$ particles are

$$
\rho_{0 n n^{\prime}}=\left(1-\epsilon e^{-x}\right)^{\epsilon} e^{-n x} \delta_{n n^{\prime}},
$$

diagonal with the Boltzmann distribution. Here $\epsilon$ is +1 for bosons and -1 for fermions. 
Thus

$$
\begin{aligned}
n_{0} & =\sum_{n=0}^{\infty} \rho_{0 n n} n=\left(e^{x}-\epsilon\right)^{-1}, \\
S_{0} & =-\operatorname{tr}\left(\rho_{0} \ln \rho_{0}\right)=-\sum_{n=0}^{\infty} \rho_{0 n n} \ln \rho_{0 n n} \\
& =x\left(e^{x}-\epsilon\right)^{-1}-\epsilon \ln \left(1-\epsilon e^{-x}\right) \\
& =\epsilon\left(1+\epsilon n_{0}\right) \ln \left(1+\epsilon n_{0}\right)-n_{0} \ln n_{0} .
\end{aligned}
$$

The entropy $S_{3}$ in the $D O W N$ mode depends on $\rho_{1}$ as well as $\rho_{0}$, but for a given $n_{3}$, it is maximized by the thermal distribution with respect to $\widetilde{\omega}$ giving that value of $n_{3}$ (i.e., not at the same temperature as $\rho_{0}$ if $n_{3} \neq n_{0}$ ), which by the formula analogous to Eq. (17) gives the upper limit

$$
S_{3} \leq \epsilon\left(1+\epsilon n_{3}\right) \ln \left(1+\epsilon n_{3}\right)-n_{3} \ln n_{3} .
$$

Combining Eqs. (14), (17), and (18) then gives

$$
\Delta \widetilde{S} \geq f_{\epsilon}\left(n_{0}, n_{3}\right) \equiv\left(\epsilon+n_{3}\right) \ln \frac{1+\epsilon n_{0}}{1+\epsilon n_{3}}-n_{3} \ln \frac{n_{0}}{n_{3}} .
$$

Clearly $f_{\epsilon}=0$ when $n_{0}=n_{3}$, and

$$
\frac{\partial f_{\epsilon}}{\partial n_{0}}=\frac{n_{0}-n_{3}}{n_{0}\left(1+\epsilon n_{0}\right)}
$$

has the same sign as $n_{0}-n_{3}$, so $f_{\epsilon}=0$ is a global minimum. Therefore, $\Delta \widetilde{S} \geq 0$ from the effect of each mode that acts independently. modes).

The above proof is valid for both nonsuperradiant and superradiant modes. It is instructive to discuss and compare these cases in more detail. The Hawking emission formula [12] says that

$$
\begin{gathered}
n_{2}=(1-\Gamma) n_{1}+\Gamma \tilde{n}_{0} \\
\tilde{n}_{0} \equiv\left(e^{\eta x}-\epsilon\right)^{-1}=\eta n_{0}+\frac{1}{2} \epsilon(\eta-1),
\end{gathered}
$$

where $1-\Gamma$ is the fraction of a classical incident wave flux that is reflected from the IN mode to the OUT mode by the angular momentum and curvature barrier around the hole. $\Gamma$ and $\tilde{n}_{0}$ are both negative for superradiant modes (bosonic modes with $\omega-m \Omega-q \Phi<0$, i.e., $\epsilon=+1$ and $\eta=-1)$.

For modes with $\eta=+1(\omega-m \Omega-q \Phi>0)$, Eq. (22) gives $\tilde{n}_{0}=n_{0}$, so Eqs. (21) and (13) imply

$$
n_{2}=\Gamma n_{0}+(1-\Gamma) n_{1}, \quad n_{3}=(1-\Gamma) n_{0}+\Gamma n_{1},
$$




$$
n_{2}+n_{3}=n_{0}+n_{1} \text {. }
$$

This is just what one would expect from a barrier with transmission probability $\Gamma$ for each UP particle to become an OUT particle and for each IN particle to become a $D O W N$ particle. Similarly, there is a reflection probability $1-\Gamma$ for each $I N$ particle to become an OUT particle and for each UP particle to become a $D O W N$ particle.

For modes with $\eta=-1(\omega-m \Omega-q \Phi<0)$, Eq. (22) gives $\tilde{n}_{0}=-n_{0}-\epsilon$, so Eqs. (21) and (13) imply

$$
\begin{gathered}
n_{2}=n_{1}-\epsilon \Gamma\left(1+\epsilon n_{0}+\epsilon n_{1}\right), \quad n_{3}=n_{0}-\epsilon \Gamma\left(1+\epsilon n_{0}+\epsilon n_{1}\right), \\
n_{2}-n_{3}=n_{1}-n_{0} .
\end{gathered}
$$

In other words, in the absence of initial particles $\left(n_{1}=n_{0}=0\right)$, the barrier produces an expected number $n_{2}=n_{3}=-\epsilon \Gamma$ of DOWN-OUT pairs (which is positive for $\eta=-1$, since then $\epsilon$ and $\Gamma$ have opposite signs), with the $n_{2}$ particles or antiparticles exiting in the OUT mode and the $n_{3} D O W N$ antiparticles or particles, respectively, entering the hole [24]. This pair-production interpretation occurs in the the "old" [10 "near-horizon" 25] viewpoint and convention we are using for the $U P$ and $D O W N$ modes (with positive local frequencies $\widetilde{\omega}>0$ ) rather than the "new" [10] "distant-observer" 25] viewpoint and convention (which uses complex conjugate $U P$ and $D O W N$ modes and so has negative local frequencies near the horizon, $\widetilde{\omega}<0$, when $\eta=-1$ ). In our convention any initial particles or antiparticles present remain on the same side of the barrier (as given by the terms in Eq. (25) independent of $\Gamma$ ), but they also induce stimulated emission (for bosons, $\epsilon=+1$, in the terms proportional to $n_{0}+n_{1}$ ) or suppression (for fermions, $\epsilon=-1$ ) by the Pauli exclusion principle. (It is interesting to note that for fixed $\omega-m \Omega-q \Phi<0$ and fixed $\Gamma>0$, the fermion emission $n_{2}$ at constant $n_{1}$ therefore decreases with increasing temperature $T_{B H}$, which increases $n_{0}$.)

It is possible also to give a simple direct proof of the generalized second law (which does not use the inequality (6)) for the particular case in which the density matrix $\rho_{1}$ in the $I N$ mode is thermal (i.e., diagonal in the Fock number basis, with entries in a geometric sequence), though not necessarily with the same temperature as $\rho_{0}$. The corresponding $O U T$ density matrix $\rho_{2}$ in this case will also be thermal, with its temperature determined by $n_{2}$, which in turn is given by Eq. (21) [3]. (D. N. P. remembers conjecturing this around 1975 to R. P. Feynman. After initial disbelief, Feynman wrote out a one-page proof that 
night.) Then Eqs. (13), (14), and the analogues of (17) for $S_{1}$ and $S_{2}$, give

$$
\Delta \widetilde{S}=S_{2}-S_{1}-\eta x\left(n_{2}-n_{1}\right)=Q_{\epsilon}\left(n_{1}\right),
$$

where

$$
Q_{\epsilon}\left(n_{1}\right) \equiv \epsilon \ln \frac{1+\epsilon n_{2}}{1+\epsilon n_{1}}+n_{1} \ln \frac{n_{1}\left(1+\epsilon \tilde{n}_{0}\right)}{\tilde{n}_{0}\left(1+\epsilon n_{1}\right)}+n_{2} \ln \frac{\tilde{n}_{0}\left(1+\epsilon n_{2}\right)}{n_{2}\left(1+\epsilon \tilde{n}_{0}\right)} .
$$

Here Eq. (22) is used to express $\eta x$ in terms of $\tilde{n}_{0}$. Eq. (21) should be used to evaluate $n_{2}\left(n_{1}\right)$. In this special case we have an explicit expression (28) for the change of the generalized entropy in terms of the particle number $n_{1}$ at $\mathcal{I}^{-}$, with no need for the inequality (6) and a discussion of states near the horizon.

To prove $\Delta \widetilde{S} \geq 0$ directly for this case, we need to show that $Q_{\epsilon}\left(n_{1}\right) \geq 0$ for any allowed value for $n_{1}$. First we note that the function obeys the relations

$$
\begin{aligned}
Q_{\epsilon}\left(\tilde{n}_{0}\right) & =\frac{d Q_{\epsilon}}{d n_{1}}\left(\tilde{n}_{0}\right)=0, \\
\frac{d^{2} Q_{\epsilon}}{d n_{1}^{2}}\left(n_{1}\right) & =\frac{A n_{1}+B}{n_{1}\left(1+\epsilon n_{1}\right) n_{2}\left(1+\epsilon n_{2}\right)} .
\end{aligned}
$$

Here

$$
A=(1-\Gamma) \Gamma\left(1+2 \epsilon \tilde{n}_{0}\right)=(1-\Gamma) \Gamma \eta\left(1+2 \epsilon n_{0}\right), \quad B=\Gamma \tilde{n}_{0}\left(1+\epsilon \Gamma \tilde{n}_{0}\right) .
$$

The quantities

$$
1-\Gamma=|R|^{2}, \quad \Gamma \tilde{n}_{0}=n_{2}\left(n_{1}=0\right) \equiv n_{\text {vac }}, \quad 1+\epsilon \Gamma \tilde{n}_{0}=1+\epsilon n_{\text {vac }}
$$

are all nonnegative, so $B \geq 0$. For bosons, $A \geq 0$ also, so for all $n_{1} \geq 0, d^{2} Q_{\epsilon} / d n_{1}^{2}$ $\geq 0$ for them. For fermions $(\epsilon=-1), A$ is negative if (and only if) $\eta<0$, but if it is, the fermionic restriction $n_{1} \leq 1$ implies that

$$
A n_{1}+B \geq A+B=\Gamma\left(1-\tilde{n}_{0}\right)\left(1-\Gamma+\Gamma \tilde{n}_{0}\right) \geq 0,
$$

so $d^{2} Q_{\epsilon} / d n_{1}^{2} \geq 0$ for them as well.

For a nonsuperradiant mode, for which $\tilde{n}_{0} \geq 0$, Eq. (29) and the concavity of $Q_{\epsilon}\left(n_{1}\right)$ implies that $Q_{\epsilon} \geq 0$. The function $Q_{\epsilon}\left(n_{1}\right)$ reaches its minimum $Q_{\epsilon}=0$ at the point $n_{1}=\tilde{n}_{0}$, where Eq. (21) implies that $n_{2}=\tilde{n}_{0}$ as well. In other words, absorption and emission of thermal states in a nonsuperradiant mode cannot decrease the generalized entropy $\widetilde{S}$. The generalized entropy is not changed if and only if there is thermal equilibrium between these $I N$ and $O U T$ states. 
For a superradiant mode, Eq. (25) implies that $n_{2}>n_{1}$. Since the thermal entropy function (17) grows with $n$, Eq. (27) with $\eta x<0$ directly shows that $\Delta \widetilde{S}>0$. In other words, any thermal state in a superradiant $I N$ mode always increases the generalized entropy.

Finally, consider the case of a Schwarzschild black hole surrounded by thermal radiation at a slightly different temperature, $T_{\text {rad }}=T_{B H}(1-\Delta)$. Summing over all particle species, helicities, and angular momenta, and integrating over all mode frequencies $\omega=(8 \pi M)^{-1} x$, one gets a total entropy production per entropy decrease of the hole of approximately

$$
\frac{d \widetilde{S}}{-d S_{B H}} \simeq \frac{\sum \int d x x^{2} e^{x}\left(e^{x}-\epsilon\right)^{-2} \Gamma\left(1-\frac{1}{2} \Gamma\right)}{\sum \int d x x^{2} e^{x}\left(e^{x}-\epsilon\right)^{-2} \Gamma} \Delta
$$

(assuming that $\Delta$ and the interaction between different modes is small). This calculation, analogous to that [5, 6] of the entropy production of radiation into vacuum, is a refinement of Eq. (11) of Zurek [1]. He effectively took $\Gamma$ to be either 0 or 1 for each mode and so got the crude result $\Delta / 2$, though we see that the actual result is between $\Delta / 2$ and $\Delta$. The latter is what we would get once we allow the emitted radiation to thermalize with the surrounding radiation. In any case, the entropy increases (since $-d S_{B H}$ and $\Delta$ have the same sign) until the black hole reaches the temperature of the radiation, assuming the total energy is constrained to make that possible [26].

To summarize, we have proved that any emission, absorption, and/or scattering by a quasistationary semiclassical black hole cannot decrease the generalized entropy $\widetilde{S}=S_{B H}+S_{\text {rad }}$. The generalized entropy $\widetilde{S}$ remains constant only when the incident radiation in the $I N$ modes is in the same thermal state as what the black hole emits in the UP modes. This is only possible for a black hole with no superradiant modes. Such a black hole must either be uncharged and nonrotating or be surrounded by a container which suppresses superradiant modes.

Acknowledgements: The authors wish to thank Werner Israel, Kip Thorne, and Robert Wald for helpful discussions and comments. This work was supported by the Danish Natural Science Research Council, grant 11-9524-1SE, and by the Natural Sciences and Engineering Research Council of Canada. 


\section{References}

[1] J. D. Bekenstein, Nuovo Cim. Lett. 4, 737 (1972); Phys. Rev. D7, 2333 (1973); and Phys. Rev. D9, 3292 (1974).

[2] R. M. Wald, in Black Holes Physics, edited by V. De Sabbata and Zhenjiu Zhang (Kluwer, Dordrecht, 1992), p. 55.

[3] I. D. Novikov and V. P. Frolov, Physics of Black Holes (Kluwer, Dordrecht, 1989).

[4] W. H. Zurek, Phys. Rev. Lett. 49, 1683 (1982).

[5] D. N. Page, Phys. Rev. D14, 3260 (1976).

[6] D. N. Page, Phys. Rev. Lett. 50, 1013 (1983).

[7] B. W. Schumacher, Phys. Rev. Lett. 54, 2643 (1985).

[8] W. G. Unruh and R. M. Wald, Phys. Rev. D25, 942 (1982).

[9] W. H. Zurek and K. S. Thorne, Phys. Rev. Lett. 54, 2171 (1985).

[10] K. S. Thorne, W. H. Zurek, and R. H. Price, in Black Holes: The Membrane Paradigm, edited by K. S. Thorne, R. H. Price, and D. A. MacDonald (Yale University Press, New Haven, 1986), p. 280.

[11] K. S. Thorne (private communication, 1993 Jan. 4).

[12] S. W. Hawking, Comm. Math. Phys. 43, 199 (1975).

[13] For example, see H. Araki and E. H. Lieb, Commun. Math. Phys. 18, 160 (1970).

[14] For a general proof of the first law, see the recent paper by D. Sudarsky and R. M. Wald, Phys. Rev. D46, 1453 (1992).

[15] M. J. Massieu, C. R. Acad. Sci. Paris 69, 858 (1869).

[16] H. B. Callen, Thermodynamics and an Introduction to Thermostatics (Wiley, New York, 1985).

[17] M. Srednicki, "Entropy and Area" (Berkeley report LBL-33754, hepth/9303048, March 1993, to be published in Phys. Rev. Lett.).

[18] V. Frolov and I. Novikov, "Dynamical Origin of the Entropy of a Black Hole" (Nordita report NORDITA-93/45A, July 1993, submitted to Phys. Rev. D). 
[19] For recent reviews, see J. A. Harvey and A. Strominger, "Quantum Aspects of Black Holes" (University of Chicago report EFI-92-41, hep-th/9209055, Sept. 1992, to appear in the proceedings of the 1992 TASI Summer School in Boulder, Colorado); S. B. Giddings, "Toy Models For Black Hole Evaporation" (University of California at Santa Barbara report UCSBTH-92-36, hep-th/9209113, to be published in String Quantum Gravity and Physics at the Planck Energy Scale, edited by N. Sanchez, World Scientific, Singapore); D. N. Page, "Black Hole Information" (University of Alberta report Thy23-93, hep-th/9305040, May 1993, to be published in Proceedings of the 5th Canadian Conference on General Relativity and Relativistic Astrophysics, University of Waterloo, 13-15 May, 1993, edited by R. B. Mann and R. G. McLenaghan (World Scientific, Singapore, 1994); U. H. Danielsson and M. Schiffer, "Quantum Mechanics, Common Sense and the Black Hole Information Paradox" (CERN report CERN-TH.6889/93, gr-qc/9305012, May 1993); S. B. Giddings, "Black Holes and Quantum Predictability" (University of California at Santa Barbara report UCSBTH-93-16, hep-th/9306041, June 1993).

[20] D. N. Page, Phys. Rev. Lett. 44, 301 (1980).

[21] G. 't Hooft, Nucl. Phys. B256, 727 (1985); and B335, 138 (1990).

[22] L. Susskind, L. Thorlacius, and J. Uglum, "The Stretched Horizon and Black Hole Complementarity" (Stanford preprint SU-ITP-93-15, hep-th/9306069, June 1993).

[23] D. N. Page, "Information in Black Hole Radiation" (University of Alberta report Thy-24-93, hep-th/9306083, June 1993, submitted to Phys. Rev. Lett.).

[24] That is, the excitations of the DOWN mode have opposite values of the energy-at-infinity, azimuthal angular momentum, and charge from those of the excitations of the OUT mode. Therefore, the two excitations are the antiparticles of each other if particles and antiparticles are distinct for the species in question. If the species does not have distinct particles and antiparticles, as is the case for photons, then both the OUT and DOWN excitations can simply be considered as particles, though they will still have opposite values of the energy-at-infinity and azimuthal angular momentum.

[25] V. P. Frolov and K. S. Thorne, Phys. Rev. D39, 2125 (1989).

[26] S. W. Hawking, Phys. Rev. D13, 191 (1976). 